\title{
Frontal Sinusitis Management: Intact Ethmoidal Bulla Technique Vs Ethmoidal Bullectomy
}

https://doi.org/10.47210/bjohns.2021.v29i2.458

Harshavardhan N. Reddy, ${ }^{1}$ Evin Abraham, ${ }^{1}$ Chandrakiran $C,{ }^{1}$ Sanjay B Patil ${ }^{1}$

Introduction

$\underline{\text { ABSTRACT }}$

Conventional functional endoscopic sinus surgery (FESS) for frontal sinusitis treatment involves ethmoidal bullectomy, that is associated with damage to the surrounding structures. These complications can be overcome by minimally invasive FESS anterior to the ethmoidal bulla that allows ease in locating the sinus ostium, eliminating risk of injury to anterior skull base and nearby structures. This study aims to compare the efficacy of ethmoidal bullectomy versus intact ethmoidal bulla technique as an adjunct to FESS in frontal sinusitis management.

Materials and Methods

Forty patients, clinically and radiologically diagnosed with frontal sinusitis, were randomly divided into 2 groups: Group A ( $n=20$; treated with FESS keeping the ethmoidal bulla intact) and Group B ( $n=20$; treated using FESS with ethmoidal bullectomy). After detailed history and clinico-radiological examinations, diagnostic nasal endoscopy was performed, followed by FESS. Pre- and post-operative endoscopic and clinical assessment was done using Modified Lund-Kennedy Endoscopy (MLKE) Score and Sino-Nasal Outcome Test-22 (SNOT-22) Questionnaire, respectively. Data was analyzed using software $R$ version 3.6.3.

\section{$\underline{\text { Results }}$}

Significant reductions in SNOT-22 (clinical improvement) and MLKE scores (endoscopic improvement) were seen within the groups from baseline to each follow-up visit and between the successive recall visits $(p<0.001)$. The SNOT-22 and $M L K E$ scores were insignificant at any visit between the groups, along with age distribution, gender and intra-operative complications ( $p>0.05$ ). Complications were seen only in Group B.

$\underline{\text { Conclusion }}$

Both, ethmoidal bullectomy and intact ethmoidal bulla technique, when used as adjuncts to FESS, showed similar improvements in frontal sinusitis patients. However, bullectomy was associated with greater risk of intra-operative complications.

$\underline{\text { Keywords }}$

Frontal Sinus; Frontal Sinusitis; Endoscopy; Sino-Nasal Outcome Test; Ethmoid Bone

$\mathrm{F}$ rontal sinusitis refers to inflammation of the frontal sinuses, usually associated with the presence of microorganisms or irritants, resulting in overproduction of mucus and/or blockage of sinus drainage. ${ }^{1,2}$ It is potentially devastating with high frequency of intracranial complications due to anatomical intimacy of frontal sinus to anterior cerebral fossa and orbit. ${ }^{1-3}$

Most patients with frontal sinusitis can be managed with appropriate medical therapy (antibiotics, decongestants). ${ }^{4,5}$ However, in advanced cases, persisting ostial stenosis and infection may necessitate surgical intervention. ${ }^{6,7}$ The most utilized classification for frontal sinusitis management is named after Prof. Wolfgang Draf - namely Draf I, Draf II, and Draf III (endoscopic Modified Lothrop procedure) techniques. ${ }^{8}$ Sinus surgery

1 - Department of ENT, M. S. Ramaiah Medical College, $M$ S Ramaiah Nagar, Mathikere, Bengaluru, Karnataka

\section{Corresponding author:}

Dr Harshavardhan N. Reddy

email: docharshavardhan@yahoo.com 
has evolved from extra-nasal to intra-nasal approach, with functional endoscopic sinus surgery (FESS) becoming the gold standard of treatment for medically refractive disease. ${ }^{9}$ It provides excellent visualization and enhanced access to the frontal sinus, allowing targeted therapy, thus improving safety and efficacy. FESS offers reduced patient morbidity and may be performed on an outpatient basis. ${ }^{6,7,9}$

Traditional frontal sinus surgery is often associated with significant trauma and poor outcomes, because it involves excision of the anterior ethmoidal sinuses, ethmoidal bullectomy and frontal sinusotomy. ${ }^{10}$ Although it reduces sinusitis symptoms and recurrence, ethmoidal bullectomy is associated with risk of postoperative bleeding, cerebrospinal fluid (CSF) leak, intracranial injuries, and damage to eye muscles and optic nerve with bleeding into the eyes that can cause blindness. ${ }^{11,12}$

These complications can be overcome by FESS anterior to the ethmoidal bulla. ${ }^{13,14}$ Since ethmoidal bulla is a relatively constant landmark, this intact bulla technique reduces the difficulty associated with locating the ostium of the frontal sinus, eliminating the possibility of injury to the anterior skull base, anterior ethmoid artery, papyraceous lamina as well as nasal and other surrounding structures. Hence, it provides a safe, simple and minimally invasive therapeutic option for frontal sinusitis patients. ${ }^{10,11,13,14}$ The entire procedure of the intact bulla technique can be performed with a $0^{\circ}$ endoscope. The technique helps keep dissection anterior to the vulnerable lateral lamella of the cribriform plate and the anterior ethmoidal artery. Since the ethmoidal bulla and bulla lamella serve as the posterior limit of the frontal recess, it is not necessary to open the ethmoidal bulla prior to dissecting the frontal recess. ${ }^{8}$ However, the ethmoidal bullectomy approach requires angled endoscopes, for which the learning curve is steeper, which is therefore prone to many complications. However, there is a paucity of scientific literature comparing the two techniques.

Therefore, the present study aimed to compare the efficacy of ethmoidal bullectomy versus intact ethmoidal bulla technique as an adjunct to FESS in frontal sinusitis management.

\section{Materials and Methods}

This hospital-based, prospective, randomized, experimental clinical study was conducted at the Department of ENT, of a tertiary care hospital, Bangalore, Karnataka, India from November 2015 to March 2017 (18 months).

The study enrolled 40 patients, aged 15-65 years, irrespective of their gender, clinically and radiologically diagnosed with isolated frontal sinusitis or isolated frontal sinus involvement. Patients with any other sinus involvement or any malignancy or revision surgery were excluded. Applying block randomization, the included participants were equally divided into 2 groups - Group $A(n=20$, treated with FESS keeping the ethmoidal bulla intact) and Group B ( $\mathrm{n}=20$; treated using FESS with ethmoidal bullectomy technique).

Complete history as well as clinical and radiological findings were recorded for all the participants, including age, gender and CT findings. Frontal sinus opacification was recorded using the Lund-MacKay CT staging method. ${ }^{15}$ A preoperative diagnostic nasal endoscopy (DNE) was performed and scored using the Modified Lund-Kennedy Endoscopy (MLKE) Score. ${ }^{16,17}$ Preoperatively, each patient was asked to report the presence of symptoms like facial or frontal pain or pressure, nasal congestion, thick nasal discharge, and postnasal drip according to the Sino-Nasal Outcome Test-22 (SNOT-22) Questionnaire. ${ }^{18,19}$

After routine blood investigations and pre-anesthetic check-up, patients were taken up for FESS under general anesthesia. All the surgeries were performed by the same surgeon familiar with both techniques. DNE was done with a zero-degree endoscope and the findings noted. Septoplasty was done in cases with difficult access to the sinuses. Group A patients were treated using FESS with the ethmoidal bulla kept intact (FESS anterior to bulla) and Group B patients using conventional FESS where the ethmoidal bulla was removed to access the frontal sinus (FESS with bullectomy). Intra-operative complications such as anterior ethmoidal artery bleeding, lamina papyracea injury, lacrimal injury and eyelid ecchymosis was noted, if any. Postoperatively, the patients were asked to report any changes experienced by them (significant improvement, mild improvement, 
no change or worsening of symptoms) using the SNOT22 Questionnaire. ${ }^{18,19}$ They were then followed at 6 weeks and 3 months postoperatively. At each followup visit, frontal sinus outflow patency was assessed using $30^{\circ} / 70^{\circ}$ rigid endoscopes. Besides the structural appearance, the patency of the frontal sinus was verified by trans-illumination (producing a bright red signal on the patient's forehead). Postoperative DNE was done and scored using the MLKE Score. ${ }^{16,17}$ The study procedure is summarized in Figure 1.

Data was compiled and analyzed using statistical software $\mathrm{R}$ version 3.6.3. Descriptive and inferential statistical analyses were carried out. Categorical variables were analyzed using Chi-square/Fisher exact test and represented as numbers (\%). Continuous variables were analyzed by performing Student t-test/ Mann Whitney $U$ test and represented as mean \pm standard deviation. A p-value $\leq 0.05$ was considered statistically significant.

\section{Results}

The study consisted of 40 frontal sinusitis patients, of whom $18(45 \%)$ were females and $22(55 \%)$ were males $(\mathrm{M}: \mathrm{F}=1.22: 1)$, with a mean age of $41.55 \pm 13.06$ years. Majority of patients (75\%) had bilateral disease. A few

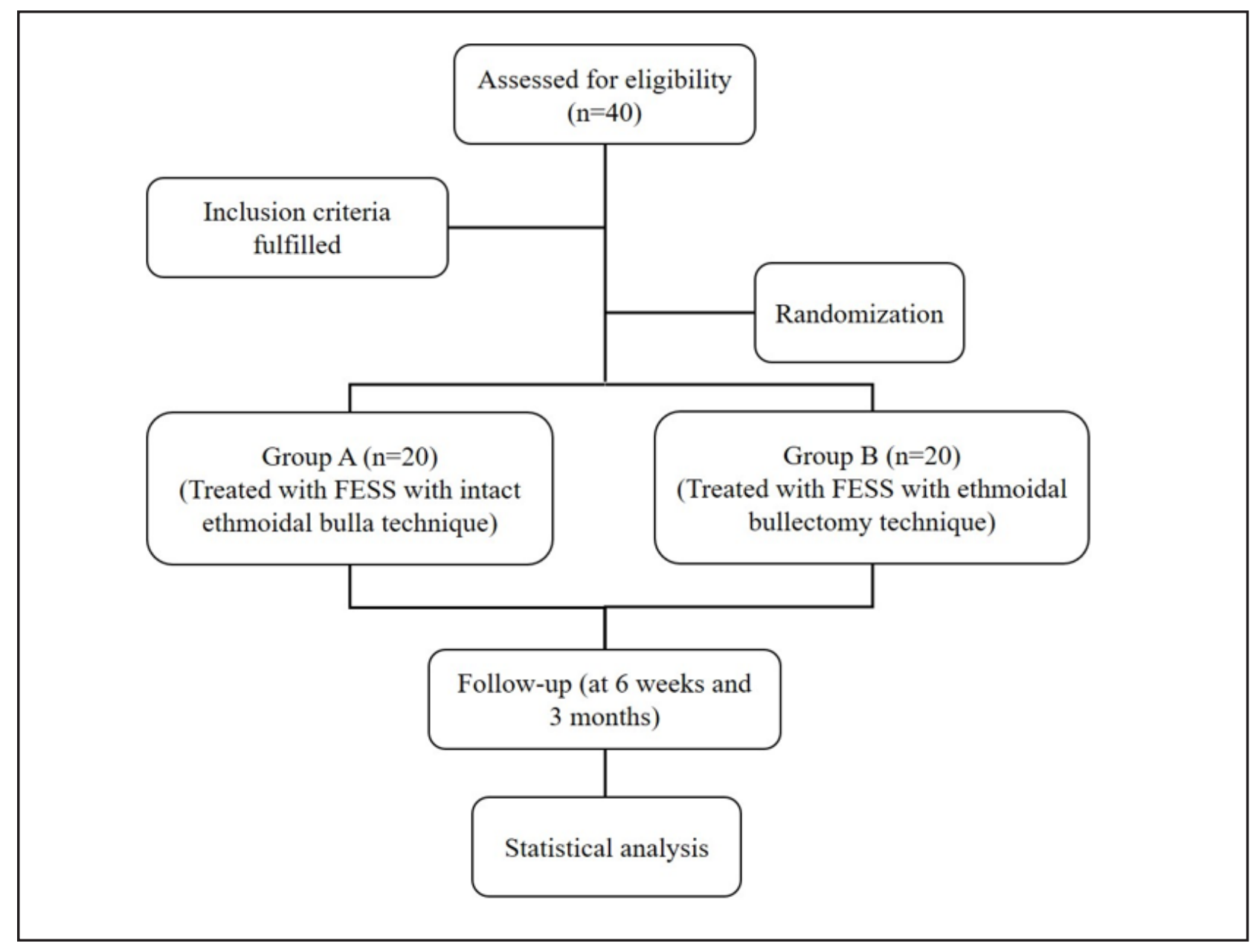

Fig. 1. Flow chart of study design 
Table I: Gender-wise frequency distribution of the various parameters

\begin{tabular}{|c|c|c|c|c|c|}
\hline \multirow{2}{*}{\multicolumn{2}{|c|}{ PARAMETERS }} & \multicolumn{3}{|c|}{ NO. OF PATIENTS (\%) } & \multirow{2}{*}{ P VALUE } \\
\hline & & FEMALE $(\mathbf{N}=18)$ & MALE $(\mathbf{N}=22)$ & TOTAL $(\mathrm{N}=\mathbf{4 0})$ & \\
\hline \multirow{6}{*}{ Age (years) } & $<\mathbf{2 0}$ & $\mathbf{0}$ & $2(9.1)$ & $2(5)$ & \multirow{6}{*}{$0.018 F^{*}$} \\
\hline & $20-30$ & $4(22.22)$ & $1(4.54)$ & $5(12.5)$ & \\
\hline & $31-40$ & $6(33.33)$ & $9(40.9)$ & $15(37.5)$ & \\
\hline & $41-50$ & $2(11.11)$ & $6(27.3)$ & $8(20)$ & \\
\hline & $51-60$ & $5(27.78)$ & $\mathbf{0}$ & $5(12.5)$ & \\
\hline & $>60$ & $1(5.56)$ & $4(18.2)$ & $5(12.5)$ & \\
\hline \multirow{3}{*}{$\begin{array}{c}\text { Side } \\
\text { involved }\end{array}$} & Bilateral & $11(61.11)$ & $19(86.36)$ & $30(75)$ & \multirow{3}{*}{$0.093 F$} \\
\hline & Left & $5(27.78)$ & $1(4.54)$ & $6(15)$ & \\
\hline & Right & 2 (11.11) & $2(9.1)$ & $4(10)$ & \\
\hline \multirow{2}{*}{ Septoplasty } & No & $13(72.22)$ & $17(77.3)$ & $30(75)$ & \multirow{2}{*}{$0.714 \mathrm{C}$} \\
\hline & Yes & $5(27.78)$ & $5(22.72)$ & $10(25)$ & \\
\hline
\end{tabular}

NOTE: F implies Fisher Exact test and C implies Chi-square test

*signifies $P$ value $<0.05$

patients $(25 \%)$ had to undergo septoplasty along with FESS for adequate visualisation of and access to the operative field. (Table I)

Table II summarizes the intergroup comparison of demographic characteristics and intra-operative complications. No significant differences were seen between the groups in the distribution of age, gender and complications $(\mathrm{p}>0.05)$, although complications were seen only in Group B but not in Group A. Lamina papyracea injury was the most common complication seen in Group B (20\%) followed by anterior ethmoidal artery bleeding (10\%) and eyelid ecchymosis (5\%).

Table III presents the frequency distribution of SNOT-22 and MLKE scores. A significant improvement of $92.5 \%$ was seen in SNOT-22 score $(p<0.001)$ and $65 \%$ in MLKE score $(\mathrm{p}<0.001)$ from pre-operative to 3 -months post-operative visit.

The mean SNOT-22 scores at pre-operative, 6-weeks post-operative and 3-months post-operative visit were $60.55 \pm 14.61,10.35 \pm 3.10$ and $8.65 \pm 2.88$ respectively. This showed a trend towards significant improvement in the disease symptoms. Intra- and inter-group comparisons of SNOT-22 and MLKE scores are presented in Table IV. Student t-test (paired) revealed significant reductions in SNOT-22 scores (clinical improvement) and MLKE scores (endoscopic improvement) within both the groups from baseline to each follow-up visit and between the successive recall visits $(p<0.001)$. However, student t-test (unpaired) found no significant difference between the groups in the mean values of SNOT-22 and MLKE scores at any visit.

\section{Discussion}

This study was conducted to compare the efficacy of ethmoidal bullectomy versus intact ethmoidal bulla technique as an adjunct to FESS in frontal sinusitis 
Table II: Intergroup comparison of demographic characteristics and intra-operative complications

\begin{tabular}{|c|c|c|c|c|}
\hline \multirow[t]{2}{*}{ PARAMETERS } & & \multicolumn{2}{|c|}{ NO. OF PATIENTS (\%) } & \multirow{2}{*}{$\begin{array}{c}\text { P VALUE } \\
\text { C/F }\end{array}$} \\
\hline & & GROUPA $(\mathrm{N}=\mathbf{2 0})$ & GROUP B (N=20) & \\
\hline \multirow{6}{*}{ Age (years) } & $<20$ & $1(5)$ & $1(5)$ & \multirow{6}{*}{0.961} \\
\hline & 20-30 & $3(15)$ & $2(10)$ & \\
\hline & $31-40$ & $7(35)$ & $8(40)$ & \\
\hline & $41-50$ & $5(25)$ & $3(15)$ & \\
\hline & $51-60$ & $2(10)$ & $3(15)$ & \\
\hline & $>60$ & $2(10)$ & $3(15)$ & \\
\hline \multirow{2}{*}{ Gender } & Female & $9(45)$ & $9(45)$ & \multirow{2}{*}{1} \\
\hline & Male & $11(55)$ & $11(55)$ & \\
\hline \multirow{4}{*}{ Complication } & $\begin{array}{c}\text { Anterior ethmoidal artery } \\
\text { bleeding }\end{array}$ & 0 & $2(10)$ & 0.106 \\
\hline & Lamina papyracea injury & $\mathbf{0}$ & $4(20)$ & 0.106 \\
\hline & Lacrimal injury & $\mathbf{0}$ & $\mathbf{0}$ & 1 \\
\hline & Eyelid ecchymosis & 0 & $1(5)$ & 1 \\
\hline
\end{tabular}

NOTE: C/F implies p-value by Chi-square test/Fisher Exact test

Table III: Frequency distribution of Sino-Nasal Outcome Test-22 and Modified Lund-Kennedy Endoscopy scores

\begin{tabular}{|c|c|c|c|c|c|}
\hline \multirow[b]{2}{*}{ PARAMETERS } & \multirow[b]{2}{*}{ SCORE } & \multicolumn{3}{|c|}{ NO. OF PATIENTS (\%) } & \multirow[b]{2}{*}{$\%$ CHANGE } \\
\hline & & $\begin{array}{c}\text { PRE- } \\
\text { OPERATIVE }\end{array}$ & $\begin{array}{l}\text { 6W POST- } \\
\text { OPERATIVE }\end{array}$ & 3M POST- & \\
\hline \multirow{5}{*}{ SNOT-22 } & $22-40$ & $3(7.5)$ & $40(100)$ & $40(100)$ & 92.5 \\
\hline & $41-60$ & $9(22.5)$ & $\mathbf{0}$ & $\mathbf{0}$ & -22.5 \\
\hline & $61-80$ & $25(62.5)$ & $\mathbf{0}$ & $\mathbf{0}$ & -62.5 \\
\hline & $81-100$ & $3(7.5)$ & $\mathbf{0}$ & $\mathbf{0}$ & -7.5 \\
\hline & $101-110$ & $\mathbf{0}$ & $\mathbf{0}$ & $\mathbf{0}$ & $\mathbf{0}$ \\
\hline \multirow{3}{*}{ MLKE } & $1-4$ & $14(35)$ & $38(95)$ & $40(100)$ & 65 \\
\hline & $5-8$ & $25(62.5)$ & $2(5)$ & $\mathbf{0}$ & -62.5 \\
\hline & 9-12 & $1(2.5)$ & $\mathbf{0}$ & 0 & -2.5 \\
\hline
\end{tabular}

NOTE: SNOT-22 = Sino-Nasal Outcome Test-22 score; MLKE = Modified Lund-Kennedy Endoscopy Score; $6 \mathrm{~W}=6$ weeks; $3 M=3$ months 
Table IV: Intra- and inter-group comparison of Sino-Nasal Outcome Test-22 and Modified Lund-Kennedy Endoscopy scores

\begin{tabular}{|c|c|c|c|c|c|}
\hline & $\begin{array}{c}\text { OPERATING } \\
\text { TIME }\end{array}$ & GROUPA & GROUP B & TOTAL & $\begin{array}{l}\text { P-VALUE } \\
\text { (1) }\end{array}$ \\
\hline \multicolumn{6}{|c|}{ SNOT-22 } \\
\hline \multirow{3}{*}{ Score $($ Mean \pm SD) } & Pre & $61.60 \pm 14.25$ & $59.50 \pm 15.24$ & $60.55 \pm 14.61$ & $0.655 t$ \\
\hline & 6w post & $9.70 \pm 2.99$ & $11.00 \pm 3.15$ & $10.35 \pm 3.10$ & $0.189 t$ \\
\hline & $3 \mathrm{~m}$ post & $8.40 \pm 2.87$ & $8.90 \pm 2.94$ & $8.65 \pm 2.88$ & $0.589 t$ \\
\hline \multirow{3}{*}{ Difference } & Pre vs 6w post & 51.9 & 48.5 & 50.2 & $0.65 t$ \\
\hline & Pre vs $3 \mathrm{~m}$ post & 53.2 & 50.6 & 51.9 & $0.18 t$ \\
\hline & $6 \mathrm{w}$ vs $3 \mathrm{~m}$ post & 1.3 & 2.1 & 1.7 & $0.58 t$ \\
\hline \multirow{3}{*}{$p$-value (2) $t$} & Pre vs 6w post & $<0.001 *$ & $<0.001 *$ & $<0.001 *$ & - \\
\hline & Pre vs $3 \mathrm{~m}$ post & $<0.001 *$ & $<0.001 *$ & $<0.001 *$ & - \\
\hline & 6w vs $3 \mathrm{~m}$ post & $<0.001 *$ & $<0.001 *$ & $<0.001 *$ & - \\
\hline \multicolumn{6}{|c|}{ MLKE } \\
\hline \multirow{3}{*}{ Score $($ Mean \pm SD) } & Pre & $5.85 \pm 2.91$ & $6.80 \pm 1.99$ & $6.33 \pm 2.51$ & $0.204 \mathrm{MW}$ \\
\hline & 6w post & $2.35 \pm 1.14$ & $2.75 \pm 1.25$ & $2.55 \pm 1.20$ & $0.31 \mathrm{MW}$ \\
\hline & $3 m$ post & $0.90 \pm 0.64$ & $1.25 \pm 0.72$ & $1.08 \pm 0.69$ & $0.108 M W$ \\
\hline \multirow{3}{*}{ Difference } & Pre vs 6w post & 3.5 & 4.05 & 3.775 & $0.41 \mathrm{MW}$ \\
\hline & Pre vs $3 \mathrm{~m}$ post & 4.95 & 5.55 & 5.25 & $0.53 \mathrm{MW}$ \\
\hline & 6w vs $3 \mathrm{~m}$ post & 1.45 & 1.5 & 1.475 & $0.706 \mathrm{MW}$ \\
\hline \multirow{3}{*}{ p-value(2) MW } & Pre vs 6w post & $<0.001 *$ & $<0.001 *$ & $<0.001 *$ & - \\
\hline & Pre vs 3m post & $<0.001 *$ & $<0.001 *$ & $<0.001 *$ & - \\
\hline & $6 w$ vs $3 \mathrm{~m}$ post & $<0.001 *$ & $<0.001 *$ & $<0.001 *$ & - \\
\hline
\end{tabular}

NOTE: SNOT-22 = Sino-Nasal Outcome Test-22 score; MLKE = Modified Lund-Kennedy Endoscopy Score; Pre = Pre-operative; $6 \mathrm{w}$ post $=6$ weeks post-operative; $3 \mathrm{~m}$ post $=3$ months post-operative; $p$-value(1) implies inter group comparison; $p$-value(2) implies intra group comparison; $t$ implies Student $t$-test and MW implies Mann Whitney U test

*signifies $P$ value $<0.05$

management. The SNOT-22 was used to assess patient-perceived changes in symptoms and quality of life. ${ }^{18,19}$ The MLKE scoring system was used to assess endoscopic changes and correlating them to clinical outcome measures. ${ }^{16,17}$ While both the techniques provided similar improvements, bullectomy was found to be associated with a greater incidence of intraoperative complications, although this difference was not statistically significant. Similar to the present study, $\mathrm{Ji}$ et $\mathrm{al}^{10}$ found complete resolution of isolated frontal sinusitis with the anterior-to-ethmoidal bulla surgical approach, and Abuzeid et $\mathrm{al}^{20}$ with the ethmoidal 
bullectomy technique.

The rationale behind success of ethmoidal bullectomy is that the frontal sinus drains dependently into the frontal recess, and hence, endoscopic procedures that avoid instrumentation of the frontal recess itself but address distal obstruction by removal of anterior ethmoid cells would resolve frontal sinus disease. ${ }^{20}$ However, it can be associated with damage to nasal, orbital and intracranial structures leading to epiphora with nasolacrimal duct injury, hemorrhage with anterior ethmoidal artery injury, periorbital ecchymosis with compromise of the lamina papyracea, restricted eye movements due to medial rectus injury, blindness due to optic nerve trauma and CSF leak due to anterior cranial base injury. ${ }^{21-23}$ This is in line with the present study where ethmoidal bullectomy technique was found to be associated with lamina papyracea injury $(20 \%)$, anterior ethmoidal artery bleeding (10\%) and eyelid ecchymosis $(5 \%)$.

The rationale behind preserving the integrity of ethmoidal bulla lies in the resection of top part of uncinate process and ager nasi cells being the key to the operation, rather than ethmoidectomy, in order to open frontal sinus drainage channel. ${ }^{10,13,24}$ Landsberg et $\mathrm{al}^{11}$ also concluded that chronic isolated frontal sinusitis develops secondary to frontal recess inflammatory changes and can be surgically treated by a targeted endoscopic procedure limited to the reestablishment of frontal sinus outflow. This procedure also avoids surgical complications, as seen in the present study, since the relatively constant position of the bulla in the middle turbinate served as a guide, eliminating the difficulty in localizing the frontal sinus ostium, allowing an angle of operation that does not damage the cranial base, anterior ethmoid artery, orbit and nasal structures..$^{10,13,14}$ However, Ji et al ${ }^{13}$ found 2 out of 329 cases developed eyelid ecchymosis with this procedure. They concluded that frontal sinus surgery via the route anterior to the ethmoid bulla is valid, relatively safe, and can be applied in most cases involving frontal sinus disease.

The present study also emphasizes the significance of mastering the surgical anatomy of the frontal sinus region, appropriate patient selection, complete pre-operative examination, and meticulous surgical technique, in order to achieve the desired results. ${ }^{10}$
The "above and below" technique (endoscopic frontal osteoplasty supplemented by a frontal sinus external trephination) and the balloon-assisted frontal sinusotomy (use of a balloon as a standalone technique to dilate the frontal recess or to assist with conventional FESS) are other techniques employed for frontal sinusitis management. ${ }^{8}$

However, this study has its limitations in being a single-center trial with a limited sample size, that does not account for sinusitis recurrence along with short term follow-up of patient's post-surgery. These can be overcome by multicentric, long-term, prospective studies with a larger sample size. Involving other management techniques could provide a better clarity on the best procedure that could be employed for managing frontal sinusitis in majority of the population.

\section{Conclusion}

Though our study found no statistical significance between ethmoidal bullectomy and intact ethmoidal bulla technique, they showed similar improvements in frontal sinusitis patients when used as adjuncts to FESS. However, bullectomy was associated with greater risk of intra-operative complications but was statistically insignificant. A larger study group with a longer followup would address the limitations of the current study.

Ethical approval: The study was approved by the Institutional Ethics Committee,Ref. No.: (STD-1/ EC/074/2015).

\section{References}

1. Brook I. Acute and chronic frontal sinusitis. Curr Opin Pulm Med. 2003; 9:171-4

2. Goldberg AN, Oroszlan G, Anderson TD. Complications of frontal sinusitis and their management. Otolaryngol Clin North Am. 2001; 34:211-25

3. Kolsi N, Zrig A, Chouchène H, Bouatay R, Harrathi K, Koubaa J. Imaging of complicated frontal sinusitis. Pan Afr Med J. 2017; 26:209

4. Anon JB, Jacobs MR, Poole MD, Ambrose PG, Benninger MS, Hadley JA, et al. Antimicrobial treatment guidelines for acute bacterial rhinosinusitis. Otolaryngol Head Neck Surg. 2004; 


\section{0:1-45}

5. Kaliner M. Medical management of sinusitis. Am J Med Sci. $1998 ; 316: 21-8$

6. Metson R, Sindwani R. Endoscopic surgery for frontal sinusitis - a graduated approach. Otolaryngol Clin North Am. 2004; $37: 411-22$

7. Metson R, Gliklich RE. Clinical outcome of endoscopic surgery for frontal sinusitis. Arch Otolaryngol Head Neck Surg. 1998; 124:1090-6

8. Lal D, Hwang PH. Endoscopic techniques in frontal sinus surgery. In: Lal D, Hwang P, editors. Frontal sinus surgery. Springer, Cham; 2019; pp. 83-125.

9. Eloy P, Andrews P, Poirrier AL. Postoperative care in endoscopic sinus surgery: A critical review. Curr Opin Otolaryngol Head Neck Surg. 2017; 25:35-42

10. Ji JF, Cheng Y, Wang TY, Wu KM, Jiang MJ, Cheng W, et al. Isolated frontal sinusitis treated using an anterior-to-ethmoidal bulla surgical approach. Cell Biochem Biophys. 2014; 70:11537

11. Landsberg R, Segev Y, Friedman M, Fliss DM, Derowe A. A targeted endoscopic approach to chronic isolated frontal sinusitis. Otolaryngol Head Neck Surg. 2006; 134:28-32

12. Ethmoidectomy sinus surgery. Available at: https://www. medtronic.com/us-en/patients/treatments-therapies/sinussurgery/functional-endoscopic-sinus-surgery/ethmoidectomy. html. Accessed Mar 26, 2020

13. Ji J, Zhou M, Li Z, Wang T, Cheng Y, Wang Q. Frontal sinus surgery anterior to the ethmoid bulla. Int Surg. 2013; 98:149-55

14. Loury MC. Endoscopic frontal recess and frontal sinus ostium dissection. Laryngoscope 1993; 103:455-8

15. Lund VJ, Mackay IS. Staging in rhinosinusitis. Rhinology
$1993 ; 107: 183-4$

16. Lund VJ, Kennedy DW. Staging for rhinosinusitis. Otolaryngol Head Neck Surg. 1997; 117:S35-40

17. Psaltis AJ, Li G, Vaezeafshar R, Cho KS, Hwang PH. Modification of the Lund-Kennedy endoscopic scoring system improves its reliability and correlation with patient-reported outcome measures. Laryngoscope 2014; 124:2216-23

18. Sino-Nasal Outcome Test (SNOT-22). Available at: https:// www.canvasc.ca/pdf/SNOT22.pdf Accessed Mar 28, 2020

19. Browne J, Hopkins C, Slack R, van der Meulen J, Lund $\mathrm{V}$, Topham J, et al. On behalf of the British Association of Otorhinolaryngologists - Head and Neck Surgeons Comparative Audit Group and the Clinical Effectiveness Unit at the Royal College of Surgeons of England. The National Comparative Audit of Surgery for Nasal Polyposis and Chronic Rhinosinusitis. Available at: https://www.rcseng.ac.uk/-/media/ files/rcs/library-and-publications/non-journal-publications/ national-comparative-audit-of-surgery-for-nasal-polyposisand-chronic-rhinosinusitis.pdf Accessed Mar 26, 2020

20. Abuzeid WM, Mace JC, Costa ML, Rudmik L, Soler ZM, Kim Gs, et al. Outcomes of chronic frontal sinusitis treated with ethmoidectomy: A prospective study. Int Forum Allergy Rhinol. 2016; 6:597-604

21. Kaluskar SK. Complications in FESS. In: Endoscopic Sinus Surgery, Springer, London; 1997. pp. 79-90

22. Stankiewiez JA. Complications of endoscopic intranasal ethmoidectomy. Laryngoscope 1987; 97:1270-3

23. Stankiewiez JA. Complications in endoscopic intranasal ethmoidectomy: An update. Laryngoscope 1989; 99:686-90

24. Wormald PJ. The agger nasi cell: The key to under- standing the anatomy of the frontal recess. Otolaryngology 2003; 129:497507. 